OPEN ACCESS

Edited by:

Phil Renforth,

Heriot-Watt University,

United Kingdom

Reviewed by:

Matthias Honegger,

Perspectives Climate Research

gGmbH, Germany

James Palmer

University of Bristol, United Kingdom

*Correspondence:

Eve Tamme

eve.tamme@climateprinciples.com

Larissa Lee Beck

Ibeck@cleanairtaskforce.org

tThese authors have contributed equally to this work and share first

authorship

Specialty section:

This article was submitted to

Negative Emission Technologies,

a section of the journa

Frontiers in Climate

Received: 19 March 2021 Accepted: 01 September 2021 Published: 27 September 2021

Citation:

Tamme E and Beck LL (2021) European Carbon Dioxide Removal

Policy: Current Status and Future Opportunities. Front. Clim. 3:682882.

doi: 10.3389/fclim.2021.682882

\section{European Carbon Dioxide Removal Policy: Current Status and Future Opportunities}

\author{
Eve Tamme ${ }^{1,2 * t}$ and Larissa Lee Beck ${ }^{3,4 * \dagger}$ \\ ${ }^{1}$ Climate Principles, Tallinn, Estonia, ${ }^{2}$ Global CCS Institute, Brussels, Belgium, ${ }^{3}$ Clean Air Task Force, Washington, DC, \\ United States, ${ }^{4}$ Atlantic Council Global Energy Center, Washington, DC, United States
}

Over the past two years, the European Union, Norway, Iceland, and the UK have increased climate ambition and aggressively pushed forward an agenda to pursue climate neutrality or net-zero emissions by mid-century. This increased ambition, partly the result of the Intergovernmental Panel on Climate Change's landmark findings on limiting global warming to $1.5^{\circ} \mathrm{C}$, has also led to a renewed approach to and revitalized debate about the role of carbon capture and storage and carbon dioxide removal. With increasing climate ambition, including a mid-century climate neutrality goal for the whole European Union, the potential role of technological carbon dioxide removal (CDR) is emerging as one of the critical points of debate among NGOs, policymakers, and the private sector. Policymakers are starting to discuss how to incentivize a CDR scale-up. What encompasses the current debate, and how does it relate to CDR technologies' expected role in reaching climate neutrality? This perspective will highlight that policy must fill two gaps: the accounting and the commercialization gap for the near-term development of a comprehensive CDR policy framework. It will shine a light on the current status of negative emission technologies and the role of carbon capture and storage in delivering negative emissions in Europe's decarbonized future. It will also analyze the role of carbon markets, including voluntary markets, as potential incentives while exploring policy pathways for a near-term scale-up.

Keywords: European Union, carbon dioxide removal, policy, negative emissions, climate change, CDR, direct air capture, BECCS

\section{INTRODUCTION}

Three years ago, the Intergovernmental Panel on Climate Change released its Special Report on $1.5^{\circ} \mathrm{C}$, outlining the potential role of $\mathrm{CDR}$ in meeting global climate targets in all four of its illustrative scenarios. With the world procrastinating substantial emissions reductions, overshooting global climate targets has become more likely, necessitating the scale-up of CDR technologies to balance out difficult or impossible-to-reduce emissions in sectors like aviation and agriculture on the pathway to net-zero, and eventually draw down historical emissions. Europe's vision for climate neutrality ${ }^{1}$ was first presented in "A Clean Planet for All" communication in 2018 (European Commission, 2018a) and became the foundation of the European Green Deal a

\footnotetext{
${ }^{1}$ Climate neutrality is defined as achieving net-zero emissions of all greenhouse gases (European Commission, 2018a), as opposed to carbon neutrality target that would include only $\mathrm{CO}_{2}$ emissions.
} 
year later (European Commission, 2019). To pave the way toward climate neutrality by 2050 , the EU raised its 2030 climate target in the recent Climate Law (European Commission, 2021b), with the Fit for 55 package laying the groundwork for its implementation.

Policy is needed to enable at scale deployment of technological CDR for three key reasons. First, little progress toward reducing emissions increases the likelihood of the need for CDR. Second, technology innovation experience has shown that it will take decades to make these technologies available at scale. Third, increased climate ambition in the near term means CDR will need to be scaled sooner. Drawing up CDR policy today would bolster the EU's position as a climate leader. Moreover, by enabling early investment in technological CDR solutions and thereby progressing commercialization and lowering the cost of these technologies, EU policy can support global access to CDR in the long term.

For successful deployment of CDR technologies, the policy must fill two gaps: the commercialization and the accounting gap, as currently, the EU policy only aims to enable a few demonstration projects. The commercialization gap is the gap between a few demonstration projects and at-scale deployment with technologies able to be deployed by climate policy, such as the EU Emissions Trading System (EU ETS) only. The commercialization gap needs to be filled by policy enabling (1) cost reductions (2) $\mathrm{CO}_{2}$ transport and storage infrastructure (3) access to affordable financing and (4) compressing deployment timelines, to enable a large scaledeployment of CDR technologies (Nagabhushan et al., 2021). Addressing the accounting gap is critical to demonstrate that actual CDR is delivered and because incentive mechanisms can only be designed for quantifiable CDR approaches (Tamme, 2021).

The paper concludes that the EU provides a promising comprehensive climate policy framework where CDR is already included in some areas. These policies are likely to be able to drive CDR deployment once the technologies are commercialized via a technology-specific innovation policy. Thus, more must be done to ensure accurate accounting and that the technologies are commercialized in time to deliver on climate ambition.

\section{DEFINING CARBON DIOXIDE REMOVAL}

The IPCC defines CDR as anthropogenic activities removing $\mathrm{CO}_{2}$ from the atmosphere and durably ${ }^{2}$ storing it in geological, terrestrial, or ocean reservoirs, or in products ${ }^{3}$ (IPCC, 2018). Tanzer and Ramírez have pointed out a high variance in how existing literature interprets $\mathrm{CDR}$, and suggest a list of four

\footnotetext{
2"Durability" and "permanence" are used interchangeably in literature. In order to meet the long-term temperature goal of the Paris Agreement, $\mathrm{CO}_{2}$ should be stored out of the atmosphere for at least hundreds of years. Shorter timescale (decades) would result in captured $\mathrm{CO}_{2}$ to be released back to the atmosphere before the mitigation goals are met. The permanence of geologic $\mathrm{CO}_{2}$ storage, if managed properly, is over a thousand years (Bergman and Rinberg, 2021).

${ }^{3}$ IPCC definition includes existing and potential anthropogenic enhancement of biological or geochemical sinks and direct air capture and storage, but excludes natural $\mathrm{CO}_{2}$ uptake not directly caused by human activities.
}

criteria to determine whether a climate solution or technology can deliver greenhouse gas removal ${ }^{4}$ (Tanzer and Ramírez, 2019).

CDR can be achieved through natural and technological approaches, ranging from biomass, soils, and oceans to storage in deep geological formations. Specific approaches like biochar and bioenergy with carbon capture and storage (BECCS) can be considered a mix of natural and technological approaches. Some approaches use biomass to draw $\mathrm{CO}_{2}$ from the air; others like direct air capture with carbon storage (DACCS) and enhanced weathering remove $\mathrm{CO}_{2}$ directly from the air. While some models, such as the illustrative P1 of the IPCC 1.5 degree report (IPCC, 2018), show that climate goals may be achieved without technological CDR options, the limited progress in making substantial emissions reductions and the challenges each solution faces suggests that a mix of CDR options will be needed.

Technological CDR approaches which rely on geologic $\mathrm{CO}_{2}$ storage include three steps: (1) $\mathrm{CO}_{2}$ is captured from the atmosphere, either directly or through biomass, (2) the $\mathrm{CO}_{2}$ is compressed and transported to the location of geologic storage, and (3) $\mathrm{CO}_{2}$ is injected to geologic formations for safe and permanent storage.

This perspective focuses mainly on the technological approaches of CDR-BECCS, and DACCS - in the context of the policy design under the European Green Deal.

\section{THE EMERGING DEBATE ON THE ROLE OF CARBON DIOXIDE REMOVAL}

The climate neutrality vision proposed in the European Green Deal and adopted in the Climate Law has substantially increased the interest in CDR. The indispensable role of CDR in achieving climate neutrality in Europe and potentially net negative emissions thereafter has led countries, corporations, cities, and regions to learn more about a range of CDR approaches, including BECCS and DACCS.

Stakeholders have voiced concerns that if emission reductions are not prioritized, CDR as a flexibility mechanism in getting to net-zero could delay climate action and water down the mitigation ambition (Carton et al., 2020). There is no common understanding of CDR's role and at least three different rationales are frequently put forward for considering CDR in public policy: (a) balancing out residual emissions from effectively-impossible-to-decarbonize sectors (like agriculture) for achieving a permanent steady state of net-zero emissions, (b) temporarily balancing out residual emissions from hardto-decarbonize sectors (like construction, heavy industry, and heavy transport), while solutions for these sectors are being developed and just transformations with job-transitions are taking place, and/or (c) to return to historical $\mathrm{CO}_{2}$ concentrations through a phase of global net-negative emissions

\footnotetext{
${ }^{4}$ Greenhouse gas removal is a broader term compared to CDR. It covers removal of all greenhouse gases, including $\mathrm{CO}_{2}$. $\mathrm{CDR}$ is the main type of greenhouse gas removal currently explored, due to the relative abundance of $\mathrm{CO}_{2}$, its long atmospheric lifetime, and its chemical reactivity which make $\mathrm{CO}_{2}$ an appealing candidate for removal (Bergman and Rinberg, 2021).
} 
after achievement of complete decarbonization (Honegger et al., 2021b).

While it is widely accepted and evident from all mid-century net-zero pathways that emission reductions will need to be strongly prioritized over CDR, it is also clear that it takes time and effort to develop new policies and scale up removals as needed. Moreover, more research and consultations will be needed to understand the socio-environmental impacts of CDR technologies, particularly in a sustainable development context. Research has shown that even a portfolio approach of CDR options might still have potentially negative implications for SDGs. Early recognition of tradeoffs and lessons learned from other technology scale-ups need to be integrated into governance and policy frameworks, and future-oriented policy must aim to minimize negative interference of technology ambition with the achievement of the SDGs, and be designed to enhance them (Honegger et al., 2021a). To conclude, policy needs to reflect both the pressing need to deliver near-term reductions and carefully govern socio-environmental impacts, while also preparing the innovation landscape for delivering CDRs in the medium-tolong term.

Currently, the slowly emerging policy debate evidences no definitive agreement, neither on the role of CDR technologies nor the necessary scale of CDR. While discussions among policymakers are yet to delve deeper into this topic and have to find consensus, the research community has kickstarted their analysis, and suggested specific thresholds and considerations for setting separate emission reduction and CDR targets (McLaren et al., 2019; Geden and Schenuit, 2020).

\section{THE ROLE OF CDR IN CLIMATE NEUTRALITY: STATUS AND POLICY}

The European Commission has stated that "In the long run, DACCS has a real potential for technological development and could become the predominant technological option to remove $\mathrm{CO}_{2}$ from the atmosphere in an energy system dominated by cheap renewable energy and batteries" (European Commission, 2018b). Indeed, CDR technologies such as DACCS are expected to deliver significant emissions reductions on the road to climate neutrality in the European Union and globally.

On the international level, the International Energy Agency's Net-Zero Scenario, a total of $2.4 \mathrm{Gt} \mathrm{CO}_{2}$ is captured in 2050 from the atmosphere through bioenergy with $\mathrm{CO}_{2}$ capture and DACCS, of which $1.9 \mathrm{GtCO}_{2}$ is permanently stored and $0.5 \mathrm{Gt}$ $\mathrm{CO}_{2}$ is used to provide synthetic fuels in particular for aviation (IEA, 2021). In the IPCC's illustrative scenarios, cumulative technological CDR until 2100 ranges from 151 GT in P2 to 1191 GT in P4 (IPCC, 2018).

For Europe, while there is a lack of comprehensive and technology-inclusive scenarios, the scenarios analyzed in the EU's communication include up to around $250 \mathrm{mtpa}$ of $\mathrm{CO}_{2}$ being removed via DACCS and BECCS in 2050 across various scenarios (European Commission, 2018b).

With regards to technological CDR, the agenda is often aligned with carbon capture and storage technologies. However, there is no single DACCS facility operating at scale ${ }^{5}$ today, and just one in planning in the United States. There are about ten or so direct air capture pilot and demonstration plants across Europe, mostly demonstrating the Climeworks technology, with a total of 15 facilities operating globally (IEA, 2020). Other examples include the Carbfix project in Iceland, the only DACCS facility that stores the $\mathrm{CO}_{2}$ underground. Climeworks also launched its 4000 tons/year Orca facility in September 2021 in Iceland (Carbfix, 2021).

Regarding BECCS, while there are several projects underway in Europe (Carbon180, 2021), including waste-to-energy plants, the Decatur Illinois BECCS facility in the United States is the only one currently operating at scale. In Europe, Drax's bio-energy CCS power station in the UK is currently in early development. The development of pilot facilities is promising, but now the technologies need to bridge the gap to at-scale deployment. This includes the pressing need to reduce costs.

While the scientific case for scaling CDR technologies is clear, there are three reasons why policy needs to support a near-term, at-scale deployment of CDR facilities, which lays out the case for filling the commercialization and accounting gaps.

First, there is a general misconception that there is sufficient time to test current, widely-adopted decarbonization technologies as main mitigation strategies before deploying more advanced decarbonization technologies like carbon capture, removal, and storage. This misconception invokes sufficient time to have a hierarchy of decarbonization technologies, i.e., that one can deploy the cheapest and politically most favored solutions first, see if they can eliminate emissions on their own, and then proceed to deploy other technologies. However, despite repeated warnings by scientists, the systems transformation at the level required to be on track to meet climate goals has not been delivered. This increases the likelihood of an emissions overshoot, requiring CDR. Coupled with the fact that the Intergovernmental Panel on Climate Change (IPCC, 2018) demonstrated that the sooner emissions are reduced, the better the chance at fending off the worst effects of climate change, it has become increasingly clear that all decarbonization options need to be deployed as soon as possible to achieve net-zero emissions by mid-century. A technology-inclusive approach that allows for multiple different technologies would increase the chances of reaching climate goals, as it would provide for a range of decarbonization technology options, mitigating the risk of any technology failing. Moreover, against the backdrop of climate change constituting a global problem, advanced economies like the European Union need to invest in decarbonization options to bring down their cost and make them available for other regions at more affordable prices. This includes CDR technologies.

Second, considering the history of innovation of primary energy production technologies, it has taken on average 20 years for technologies to reach a $1 \%$ market penetration level which is regarded mainly as an inflection point for a technology (Kramer and Haigh, 2009). This is a deft analogy because primary energy production technologies are similar in scale, complexity, additionality, and new infrastructure

\footnotetext{
${ }^{5}$ Capturing 500,000 tons of $\mathrm{CO}_{2}$ or more.
} 
requirement. Moreover, the investment risk profiles of new technologies are similar to other clean energy technologies at early stages. At a $1 \%$ market penetration level, typically, hundreds of billions of dollars have been spent on technology development and early project deployment to reach the point of providing $1 \%$ of the world's primary energy production. Evidence suggests that it then takes about 30 additional years to get a threshold of $20-30 \%$ of primary energy demand, underlining the challenges of scaling technologies fast and within the required timeframe to achieve net-zero emissions. This provides evidence of the challenges of scaling these technologies in a short amount of time and supports the arguments of developing policies to scale them in the near term.

Third, as laid out in its new 2030 target to achieve at least a 55\% emissions reduction below 2010, the EU's increased ambition necessitates CDR technologies to be scaled sooner. As the impact assessment states: "Increased ambition increases clarity on the pace of emissions reductions required (...) increasing the role of CDRs in our economy" (European Commission, 2020b). Moreover, CDR could also be considered a pathway to deliver climate change mitigation at different speed across the Member States to reach the 2030 goal and beyond, effectively contributing toward the collective goal.

To fill the commercialization gap and considering these implications of the innovation timeframe, an EU policy framework must speed up deployment timelines through enabling key success factors, including cost reductions, $\mathrm{CO}_{2}$ networks, access to affordable investment, and compressing deployment timelines. Cost reductions are necessary to enable more effective deployment at a cheaper price and would eventually enable comprehensive climate policy such as carbon pricing to be the sole driver of technology deployment. Lower cost through deployment and learning-by-doing would also de-risk the technologies, and attract more investors, enabling more affordable capital to flow into CDR. Access to existing infrastructure such as $\mathrm{CO}_{2}$ transport and storage would also make it much easier to build additional CDR facilities.

Fortunately, it is expected that DACCS and BECCS will benefit from the overall push to deploy point-source carbon capture and storage technologies. This includes the $\mathrm{CO}_{2}$ infrastructure build-out currently underway in the Nordics and the Netherlands and is already evidenced by new partnerships built around these projects. For example, Climeworks announced a collaboration with the Northern Lights Project, a $\mathrm{CO}_{2}$ transport and storage project off Norway's coast (Farmer, 2021). The Government of Norway committed some $€ 2 \mathrm{~B}$ to the project, covering initial capital investment and 10 years of operating expenses. By lowering the cost of transport and storage cost, which can be significant through achieving economies of scale, $\mathrm{CO}_{2}$ transport and storage networks are essential enablers of CDR deployment. Moreover, Orsted, Aker Carbon Capture, and Microsoft also announced a Memorandum of Understanding to explore retrofitting one of Orsted's biomass facilities in Denmark (Ørsted, 2021).

\section{PATH FORWARD, BUILDING ON THE CURRENT POLICY LANDSCAPE}

As outlined above, the policy must serve to speed up commercialization timelines and enable the four success factors. This section will make the case that current EU policy is expected to enable some demonstration projects, but falls short of enabling at-scale deployment-evidencing the commercialization gap.

Over the following years, EU policy-making will be guided by aligning existing sectoral legislation-such as the EU ETS and the Effort Sharing Regulation-with the new 2030 and 2050 climate targets through the Fit for 55 package proposed by the European Commission in July 2021. This comprehensive climate policy reform is an opportunity to provide clear rules and policy pathways for CDR, as ultimately, the emerging policy framework described below is expected to drive the investments in CDR demonstrations in the near term.

The EU ETS currently covers around $40 \%$ of EU emissions and will be key to eventually driving investment in commercialized technologies on its own. Established as the world's largest cap and trade system, its carbon price is over $50 €$ in mid-2021.

One of the policies in the EU ETS toolbox is the EU Innovation Fund, a capital support mechanism for innovative technologies financed by auctions of EU allowances. Worth $22 \mathrm{~B} €$ at the current EU ETS allowance price, it is relatively small compared to the ambition. The Fit for 55 package proposes a doubling of its capacity and also including carbon contracts for difference (CCfDs). Innovation Fund's first round of applications was oversubscribed by a factor of 20. An analysis of the applications indicates that a handful of the submitted projects can deliver "net CDR," while around a fifth of total applications include carbon capture, utilization, and storage technologies (European Commission, 2020d). The Innovation Fund could indeed drive investment in CDR demonstration projects in Europe and form an essential risk-reduction tool to enable access to additional private capital for demonstration projects, and perhaps for further projects that could benefit from the grant and technologyspecific incentive mechanisms alike. First-mover demonstration projects also make outsized contributions for cost reduction opportunities. However, due to its limited size and the funds being shared across a range of technologies, it is unlikely to be sufficient for at-scale commercialization.

The Trans-European Energy Networks Regulation, also known as TEN-E, is critical for transboundary $\mathrm{CO}_{2}$ transportation networks and thus enabling $\mathrm{CO}_{2}$ infrastructure success factors. TEN-E establishes criteria for projects (Projects of Common Interest) that have access to a list of benefits, including access to funding from the Connecting Europe Facility. In the ongoing revision, the European Commission proposed to continue including cross-border $\mathrm{CO}_{2}$ pipelines in the scope of TEN-E while resisting stakeholder requests to include the entire value chain of carbon capture and storage, including alternative $\mathrm{CO}_{2}$ transport options such as rail, barge, ship, and truck, along with geologic $\mathrm{CO}_{2}$ storage (European Commission, 2020c). CDR advocates argue that a broader inclusion of $\mathrm{CO}_{2}$ infrastructure would provide more certainty 
for project development. It would further incentivize long-term investment by demonstrating government commitment to the necessary infrastructure.

Establishing a well-designed cross-border $\mathrm{CO}_{2}$ infrastructure in Europe is especially relevant in the context where the access $\mathrm{CO}_{2}$ storage is not distributed evenly among countries. The hubs and cluster design of several CCS projects in development illustrates the need for $\mathrm{CO}_{2}$ to cross several country borders to be transported to storage sites. It helps address the chicken and egg problem; capture carbon is needed to invest in the infrastructure to transport and store it, but firms are unlikely to support investment in capture without this infrastructure. Furthermore, geologic storage inclusion would alleviate the inequitable distribution of geologic storage resources among member states (Pozo et al., 2020). This argument becomes particularly relevant regarding technological CDR, as it is only permanent when coupled with geologic storage while providing transnational benefits of lowering the overall $\mathrm{CO}_{2}$ concentration already in the atmosphere.

Overall, the currently existing European policy framework, with expected and necessary revisions forthcoming, is wellestablished to enable initial demonstration projects and anchor infrastructure to be built and offers promising opportunities for integrating incentive mechanisms for CDR.

\section{FILLING THE ACCOUNTING AND COMMERCIALIZATION GAPS}

To commercialize CDR technologies, two gaps must be filled by policy and regulation: the accounting gap and the commercialization gap.

Addressing the accounting gap is critical to demonstrate that actual CDR is delivered and because incentive mechanisms can only be designed for quantifiable CDR approaches (Tamme, 2021). This goes for CDR in general and more specifically in the context of the newer CDR approaches, including DACCS, that are not currently covered by sectoral climate policies. Below the authors provide an outline of opportunities for improvement in existing policies and pathways to improve the policy framework.

To address the accounting gap, the European Commission is preparing a regulatory framework carbon removal certification (CRC) to be proposed by 2023 (European Commission, 2020a). Commission communication on restoring sustainable carbon cycles, expected at the end of 2021, will "identify key elements to build a robust and credible framework allowing for authentic, transparent and verifiable carbon removals to be certified" (European Commission, 2021c). Preparation of this policy faces a two-fold challenge: (1) CRC should meaningfully incentivize the deployment of CDR approaches while (2) also supporting the notion of prioritizing emission reductions over removals, especially in the decades leading up to climate neutrality. Different levels of permanence among CDR approaches, coupled with challenging monitoring, reporting, and verification when it comes to nature-based approaches, will add to the complexity. Developing robust accounting rules that can be used to design policies for incentivizing CDR will also facilitate the commercialization of CDR technologies. The preparatory work on the CRC framework has already started, and it is expected to become operational in 2024-2025.

To address the commercialization gap, the policy must enable the four success factors to be met, while providing a long-term incentive for continued investment to build multiple facilities beyond just demonstration projects.

Policy options that could work on the Member State level include policies addressing upfront investment barriers and both CAPEX and OPEX economics of CDR projects. Many of the US projects under development received grants for feasibility and front-end-engineering design (FEED) studies (Beck, 2020) (Zapantis et al., 2019). While the initial public investment is relatively low-in the order of millions-such FEED study grants can help overcome initial barriers to investment by covering upfront cost even if the outcome is uncertain. To address financing gaps and draw in traditional financing, capital grants could increase certainty and demonstrate government commitment. These could be additional to capital grants to those offered through the EU Innovation Fund.

Moreover, CCfDs are gaining traction across Europe as incentive mechanisms for next-generation clean energy technologies, such as hydrogen. Their imperative is bridging the gap between the actual cost of decarbonization technologies and the price of a benchmark, i.e., the EU ETS if coupled with geologic storage of $\mathrm{CO}_{2}$. The rationale for CCfDs is that the $\mathrm{EU}$ ETS carbon price is not high enough to incentivize technology uptake without complementary innovation policies. They have successfully supported the commercialization of renewable energy technologies in the form of feed-in tariffs, thus poised to integrate innovation objectives vis-à-vis existing climate policy. The new proposal for the revision of the EU ETS suggests CCfDs as part of the Innovation Fund (European Commission, 2021a). Moreover, specific instruments are discussed in several Member States. The Dutch SDE++ closed its first round of funding for decarbonization technologies. It offers a 15-year CCfD for the delta between an agreed price and the EU ETS carbon price. These mechanisms can generate sufficient funding to enable multiple projects.

While policymakers need to flesh out how to fill the commercialization gap, other mechanisms could help with project economics to accelerate the deployment of CDR technologies in Europe. First is the not well-known California Low Carbon Fuel Standard CCS Protocol. Second is carbon pricing, including compliance and voluntary carbon markets.

Counterintuitively, California's Low Carbon Fuel Standard (LCFS) incentivizes DACCS investment anywhere globally, including Europe. Trading at around $\$ 200 / \mathrm{t}$ of $\mathrm{CO}_{2}$, the LCFS aims to reduce the emissions intensity of fuels consumed in California by $20 \%$ by 2030 . Recognizing that $\mathrm{CO}_{2}$ emissions are a global problem, the LCFS incentivizes DACCS projects anywhere in the world, as long as they adhere to the LCFS CCS protocol's rules (Townsend and Havercroft, 2019). Working with private-sector stakeholders to deliver projects under the California LCFS might also open international collaboration opportunities on innovation and knowledge sharing (Beck and Livingston, 2019). However, policymakers 
should ensure accurate accounting, potentially aligning different policies.

DACCS and BECCS have also gained a lot of interest in the voluntary carbon markets since 2020 as over a thousand companies have already set net-zero targets. Currently, there are no methodologies for DACCS and BECCS projects under the major voluntary market standards ${ }^{6}$ and the transactions take place outside the main standards. Some examples from Europe include Climeworks from Switzerland selling subscriptions for CDR from the air and the Puro.earth CDR marketplace in Finland offering a range of $\mathrm{CDR}$ products and preparing a methodology for BECCS and geologically stored carbon (Puro.earth, 2021).

Given that the current Nationally Determined Contributions under the Paris Agreement fall well short of the 2C target (let alone the 1.5C) (UNEP, 2020), the voluntary markets could play a role in bridging the mitigation gap to achieve the Paris Agreement temperature goal. However, the potential overlap in activities under compliance and voluntary markets, such as potential double claiming of emission reductions or removals, must be carefully tackled. As an example, more than 10 companies in Sweden are planning to implement BECCS between 2025 and 2030 (Schenuit et al., 2021). Some of these BECCS operators intend to supply removal credits to voluntary as well as compliance markets (Fridahl and Lundberg, 2021). Hence, both the governments and voluntary market actors will need to work together to ensure that double claiming risks are mitigated.

\section{MOVING FORWARD: POLICY DESIGN FOR SCALING UP CDR UNDER THE EUROPEAN GREEN DEAL}

This perspective aimed to give an overview of CDR technology and policy in the European Union. CDR technology commercialization is necessary in the near term because (1) the world will likely overshoot its climate goals-and is expected to do so as a global collective-highlighting the importance of investing in a diverse portfolio of decarbonization options as soon as possible. (2) History has shown that the

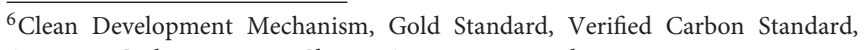
American Carbon Registry, Climate Action Reserve, Plan Vivo.

\section{REFERENCES}

Beck, L. (2020). Carbon capture and storage in the USA: the role of US innovation leadership in climate-technology commercialization. Clean Energy 4, 2-11. doi: $10.1093 / \mathrm{ce} / \mathrm{zkz031}$

Beck, L., and Livingston, D. (2019). “The California climate policy no one is talking about," in The Hill. Available online at: https://thehill.com/opinion/energyenvironment/451526-the-california-climate-policy-no-one-is-talking-bout.

Bergman, A., and Rinberg, A. (2021). “The Case for Carbon Dioxide Removal: from Science to Justice," in CDR Primer, eds J. Wilcox, B. Kolosz, J. Freeman. Available online at: https://cdrprimer.org/read/chapter-1.

Carbfix (2021). The worlds first direct air $\mathrm{CO}_{2}$ capture and storage plant is ON. Available online at: https://www.carbfix.com/former-president-oficeland-mr.-olafur-ragnar-grimsson commercialization of technologies takes several decades and can only be accelerated with adequate policy, which in itself has multi-year led times, and (3) higher EU climate ambition increases the importance of CDR technologies, both to address hard-to-abate sectors and deliver net-negative emissions, but there are two significant gaps: commercialization and accounting.

Yet, a policy must be designed to deliver on technology and climate ambition, including cost reduction, drawing in affordable finance, addressing $\mathrm{CO}_{2}$ geologic storage and transport infrastructure needs, and accelerating deployment timelines. While the EU already has robust climate policy frameworks amenable to the inclusion of CDR incentives, there is an innovation policy gap that needs to be bridged to enable the large-scale commercialization of CDR technologies. There are several ways this gap could be filled, including through CCfDs and other CAPEX mechanisms and establishing robust greenhouse gas accounting for CDR approaches, incentivized by carbon removal certification framework.

The role of emission reductions and removals in the mitigation of climate change will change over time. Emission reductions will be prioritized on the path to net-zero. However, net-zero by mid-century is a point on the journey to addressing the climate crisis, not the final goal. Thus, CDR will become the main driver to deliver on climate ambition in the second half of the century.

\section{DATA AVAILABILITY STATEMENT}

The original contributions presented in the study are included in the article, further inquiries can be directed to the corresponding authors.

\section{AUTHOR CONTRIBUTIONS}

All authors listed have made a substantial, direct and intellectual contribution to the work, and approved it for publication.

\section{ACKNOWLEDGMENTS}

The authors would like to thank Alex Zapantis from the Global CCS Institute for his review of the first draft of this piece.

Carbon180 (2021). Carbon180 DAC map. Available online at: https://carbon180. org/dac-mapp (accessed March 12, 2021).

Carton, W., Asiyanbi, A., Beck, S., Buck, H. J., and Lund, J. F. (2020). "Negative emissions and the long history of carbon removal," in Wiley Interdisciplinary Reviews: Climate Change, 1-25.

European Commission (2018a). “A Clean Planet for all. A European long-term strategic vision for a prosperous, modern, competitive and climate neutral economy," COM(2018) 773 final. Available online at: https://eur-lex.europa.eu/ legal-content/EN/TXT/?uri=CELEX:52018DC0773.

European Commission (2018b). In-depth analysis in support of the Commission communication COM(2018) 773. A Clean Planet for all. A European longterm strategic vision for a prosperous, modern, competitive and climate neutral economy. Available online at: https://ec.europa.eu/clima/sites/clima/files/docs/ pages/com_2018_733_analysis_in_support_en_0.pdf. 
European Commission (2019). The European Green Deal. COM(2019) 640 final. Available online at: https://eur-lex.europa.eu/legal-content/EN/TXT/? qid $=157615054271$ anduri=COM\%3A2019\%3A640\%3AFIN.

European Commission (2020a). A new Circular Economy Action Plan For a cleaner and more competitive Europe. $\operatorname{COM}(2020) 98$ final. Available online at: https://eur-lex.europa.eu/legal-content/EN/TXT/PDF/?uri=CELEX: 52020DC0098andfrom=EN.

European Commission (2020b). Impact Assessment, accompanying Communication'Stepping up Europe's 2030 climate ambition-Investing in a climate-neutral future for the benefit of our people. SWD(2020) 176 final. Available online at: https://ec.europa.eu/clima/sites/clima/files/eu-climateaction/docs/impact_en.pdf.

European Commission (2020c). Proposal for a Regulation of the European Parliament and of the Council on guidelines for trans-European energy infrastructure and repealing Regulation (EU) No 347/2013. Available online at: https://eur-lex.europa.eu/resource.html?uri=cellar:cc5ea219-3ec7-11ebb27b-01aa75ed71a1.0002.02/DOC_1andformat=PDF.

European Commission (2020d). Statistics of the proposals received for the first large-scale call of the Innovation Fund in October 2020. Available online at: https://ec.europa.eu/clima/sites/default/files/innovation-fund/largescale_call_statistics_en.pdf.

European Commission (2021a). Proposal for a DIRECTIVE OF THE EUROPEAN PARLIAMENT AND OF THE COUNCIL amending Directive 2003/87/EC establishing a system for greenhouse gas emission allowance trading within the Union, Decision (EU) 2015/1814 concerning the establishment and operation o. Available at: https://eur-lex.europa.eu/legal-content/EN/TXT/?uri=CELEX: 52021PC0551.

European Commission (2021b). REGULATION (EU) 2021/1119 OF THE EUROPEAN PARLIAMENT AND OF THE COUNCIL of 30 June 2021 establishing the framework for achieving climate neutrality and amending Regulations (EC) No 401/2009 and (EU) 2018/1999 ('European Climate Law'), Official Journal of the European Union. Available online at: https://eur-lex. europa.eu/eli/reg/2021/1119/oj.

European Commission (2021c). Roadmap. Restoring sustainable carbon cycles. Available online at: https://ec.europa.eu/info/law/better-regulation/have-yoursay/initiatives/13066-Climate-change-restoring-sustainable-carbon-cycles_en

Farmer, M. (2021). "Northern Lights CCS project to explore direct air capture," in Offshore Technology. Available online at: https://www.offshore-technology. $\mathrm{com} /$ news/company-news/northern-lights-ccs-equinor-shell-total-directair-carbon-capture-negative-emissions/.

Fridahl, M., and Lundberg, L. (2021). Aktörspreferenser i design av ett stödsystem för bio-CCS. LINKÖPINGS UNIVERSITET. Available online at: https:/liu.divaportal.org/smash/get/diva2:1548418/FULLTEXT01.pdf.

Geden, O., and Schenuit, F. (2020). Unconventional Mitigation: Carbon Dioxide Removal as a New Approach in EU Climate Policy. Berlin: Stiftung Wissenschaft und Politik.

Honegger, M., Michaelowa, A., and Roy, J. (2021a). Potential implications of carbon dioxide removal for the sustainable development goals. Clim. Policy 21, 678-698. doi: 10.1080/14693062.2020.1843388

Honegger, M., Porallam, M., Michaelowa, A., and Ahonen, H.-M. (2021b). Who is paying for carbon dioxide removal? designing policy instruments for mobilizing negative emissions technologies. Front. Clim. 3:672996. doi: $10.3389 /$ fclim.2021.672996

IEA (2020). Energy Technology Perspectives 2020 - Special Report on Carbon Capture Utilisation and Storage. Paris.

IEA (2021). Net Zero by 2050. A Roadmap for the Global Energy Sector. Paris.

IPCC (2018). Global Warming of $1.5^{\circ} \mathrm{C}$. An IPCC Special Report on the impacts of global warming of $1.5^{\circ} \mathrm{C}$ above pre-industrial levels and related global greenhouse gas emission pathways, in the context of strengthening the global response to the threat of climate change. Available online at: https://www.ipcc.ch/sr15/.

Kramer, G. J., and Haigh, M. (2009). No quick switch to low-carbon energy. Nature 38, 568-569. doi: $10.1038 / 462568 \mathrm{a}$

McLaren, D. P., Tyfield, D. P., Willis, R., Szerszynski, B., and Markusson, N. O. (2019). 'Beyond "Net-Zero": a case for separate targets for emissions reduction and negative emissions. Front. Clim. 1:4. doi: 10.3389/fclim.2019. 00004

Nagabhushan, D., Russell, R. H., Waltzer, K., Thompson, J., Beck, L., and Jaruzel, M. (2021). Carbon capture: Prospects and policy agenda for CO2-neutral power generation. Electr. J. 34:106997. doi: 10.1016/j.tej.2021.106997

Ørsted (2021). Ørsted, Aker Carbon Capture, and Microsoft commit to explore development of carbon capture. Available online at: https://orsted.com/en/ media/newsroom/news/2021/03/569767710474825 (accessed March 12, 2021).

Pozo, C., Galán-Martín, Á., Reiner, D. M., Mac Dowell, N., and Guillén-Gosálbez, G. (2020). Equity in allocating carbon dioxide removal quotas. Nat. Clim. Change 10, 640-646. doi: 10.1038/s41558-020-0802-4

Puro.earth (2021). BECCS and Geologically Stored Carbon Methodology Webinar. Available online at: https://puro.earth/articles/beccs-and-geologically-storedcarbon-methodology-webinar-584 (Accessed March 12, 2021).

Schenuit, F., Colvin, R., Fridahl, M., McMullin, B., Reisinger, A., Sanchez, D. L., and Geden, O. (2021). Carbon dioxide removal policy in the making: assessing developments in 9 OECD Cases. Front. Clim. 3:638805. doi: $10.3389 /$ fclim.2021.638805

Tamme, E. (2021). Carbon removal with CCS technologies. Global CCS Institute. Available online at: https://www.globalccsinstitute.com/resources/ publications-reports-research/carbon-removal-and-ccs/.

Tanzer, S. E., and Ramírez, A. (2019). When are negative emissions negative emissions? Energy Environ. Sci. 12, 1210-1218. doi: 10.1039/C8EE03338B

Townsend, A., and Havercroft, I. (2019). The LCFS and CCS Protocol: An Overview for Policymakers and Project Developers, Global CCS Institute. Available online at: https://www.globalccsinstitute.com/resources/publications-reportsresearch/the-lcfs-and-ccs-protocol-an-overview-for-policymakers-andproject-developers/.

UNEP (2020). Emissions Gap Report 2020. Nairobi. Available online at: https:// www.unep.org/emissions-gap-report-2020.

Zapantis, A., Townsend, A., and Rassool, D. (2019). Policy Priorities To Incentivise Large Scale Deployment of CCS. Global CCS Institute. Available online at: https://www.globalccsinstitute.com/resources/publications-reportsresearch/policy-priorities-to-incentivise-large-scale-deployment-of-ccs/.

Conflict of Interest: The authors declare that the research was conducted in the absence of any commercial or financial relationships that could be construed as a potential conflict of interest.

Publisher's Note: All claims expressed in this article are solely those of the authors and do not necessarily represent those of their affiliated organizations, or those of the publisher, the editors and the reviewers. Any product that may be evaluated in this article, or claim that may be made by its manufacturer, is not guaranteed or endorsed by the publisher.

Copyright () 2021 Tamme and Beck. This is an open-access article distributed under the terms of the Creative Commons Attribution License (CC BY). The use, distribution or reproduction in other forums is permitted, provided the original author(s) and the copyright owner(s) are credited and that the original publication in this journal is cited, in accordance with accepted academic practice. No use, distribution or reproduction is permitted which does not comply with these terms. 